\title{
Reduced Hypoglycemia Risk in Type 2 Diabetes Patients Switched to/Initiating Insulin Glargine 300 vs 100 U/ml: A European Real-World Study
}

\author{
Javier Escalada Fabrice Bonnet · Jasmanda $\mathrm{Wu} \cdot$ Mireille Bonnemaire \\ Shaloo Gupta · Janelle M. Cambron-Mellott • Charlie Nicholls • \\ Dirk Müller-Wieland
}

Received: April 30, 2020 / Published online: July 17, 2020

(C) The Author(s) 2020

\section{ABSTRACT}

Introduction: Randomized controlled trials and real-world data from the USA have shown similar glycemic control with insulin glargine $300 \mathrm{U} / \mathrm{ml}$ (Gla-300) and insulin glargine 100 $\mathrm{U} / \mathrm{ml}$ (Gla-100) and reduced hypoglycemia risk with Gla-300. This real-world study describes the efficacy and safety of Gla-300 and Gla-100 in patients with type 2 diabetes (T2D) in France, Spain, and Germany.

Digital Features To view digital features for this article go to https://doi.org/10.6084/m9.figshare.12608261.

Electronic Supplementary Material The online version of this article (https://doi.org/10.1007/s12325020-01436-5) contains supplementary material, which is available to authorized users.

J. Escalada $(\square)$

Clinic University of Navarra, Pamplona, Spain

e-mail: fjavier.escalada@gmail.com

\section{J. Escalada}

Biomedical Research Networking Center for Physiopathology of Obesity and Nutrition (CIBEROBN), Institute of Health Carlos III, Pamplona, Spain

J. Escalada

Diabetes and Metabolic Diseases, Institute of Health

Research of Navarra (IdiSNA), Pamplona, Spain

F. Bonnet

CHU de Rennes, Université Rennes 1, Rennes, France
Methods: This retrospective chart review analysis used anonymized data for adults with T2D switching basal insulin analog (BIA) therapy to Gla-300 or Gla-100, or insulin-naïve patients initiating Gla-300 or Gla-100. Outcomes included change from baseline to 6-month follow-up in glycated hemoglobin A1c (A1C), total and severe hypoglycemia incidences and events, insulin dose, and reasons for BIA choice.

Results: Six hundred sixty-five physicians (33.8\% Spain, 31.7\% France, 34.4\% Germany) provided chart data for patients switching to Gla-300 $(n=679)$ or Gla-100 $(n=429)$ or initiating Gla$300(n=719)$ or Gla-100 $(n=711)$. After adjustment for baseline characteristics, $\mathrm{A} 1 \mathrm{C}$ reductions from baseline were similar for patients switching to Gla-300 or Gla-100 ( $-0.87 \%$ vs. $-0.93 \%$; $p=0.326$ ) while those switched to Gla-300 vs. Gla100 had a significantly greater mean reduction in

J. Wu

Sanofi, Bridgewater, NJ, USA

M. Bonnemaire

Sanofi, Paris, France

S. Gupta · J. M. Cambron-Mellott

Kantar, New York, NY, USA

C. Nicholls

Sanofi, Reading, UK

D. Müller-Wieland

Department of Medicine, University Hospital

Aachen, Aachen, Germany 
hypoglycemic events ( -1.29 vs. -0.81 events during 6 months; $p=0.012$ ). Mean insulin doses after titration were $0.43 \pm 0.36$ and $0.40 \pm 0.28$ $\mathrm{U} / \mathrm{kg}$ in Gla-300 and Gla-100 switchers, respectively. Factors that significantly influenced BIA choice included a lower risk of hypoglycemia (for Gla-300) and physician familiarity (for Gla-100). Outcomes for insulin-naïve patients were broadly similar to those of switchers.

Conclusions: In this real-world European study, patients with T2D who switched therapy to Gla300 or Gla-100 had improved glycemic control and reduced hypoglycemia at 6 months, with significant hypoglycemia advantages with Gla300 .

Keywords: Diabetes mellitus; Hypoglycemia; Insulin glargine; Type 2

\section{Key Summary Points}

Why carry out this study?

Randomized controlled trials and realworld comparative studies from the US have shown that insulin glargine $300 \mathrm{U} / \mathrm{ml}$ (Gla-300) provides similar glycemic control to insulin glargine $100 \mathrm{U} / \mathrm{ml}$ (Gla-100) but is less likely to cause hypoglycemia.

However, data from Europe are scarce.

This real-world study therefore described the efficacy and safety of Gla-300 and Gla100 in patients with type 2 diabetes (T2D) in Spain, France, and Germany.

\section{What was learned from the study?}

Patients with T2D who switched from another basal insulin analog to Gla-300 or Gla-100 had similarly improved glycemic control, while those who switched to Gla300 had greater reductions in hypoglycemia.

Insulin-naïve patients with T2D who initiated Gla-300 or Gla-100 also had greater reductions in hypoglycemia.

Physicians selected Gla-300 over Gla-100 to achieve a lower risk of hypoglycemia.

\section{INTRODUCTION}

Approximately 60 million people in Europe have diagnosed type 2 diabetes (T2D), meaning that this disease affects around $10 \%$ of European adults aged $\geq 25$ years [1]. Thus, T2D is a major burden on individuals and healthcare systems [2]. Joint American and European guidelines recommend lifestyle interventions as the first-line intervention, metformin as the first-line medication, and glucagon-like peptide 1 receptor antagonists as the first-line injectable medication [3]. As T2D is a progressive disease, patients will often eventually require insulin therapy [2]. However, there are a number of barriers to the initiation of insulin including fear of injections and hypoglycemia $[2,4]$.

A second-generation basal insulin analog (BIA), insulin glargine $300 \mathrm{U} / \mathrm{ml}$ (Gla-300), has been associated with significant reductions in some hypoglycemia outcomes (and comparable improvements in glycemic control) in randomized controlled trials (RCTs) in patients with T2D vs. the first-generation BIA, insulin glargine $100 \mathrm{U} / \mathrm{ml}$ (Gla-100) [5-9], which has been confirmed in recent trial-level [10] and patient-level $[11,12]$ meta-analyses. This advantage is likely due to Gla-300's improved pharmacokinetic/pharmacodynamic (PK/PD) profile, longer half-life, and more stable bioavailability [13-15]. The reduced risk of hypoglycemia, in combination with additional advantages, such as reduced injection volumes and flexible dosing times, may allow second-generation BIAs to overcome some of the barriers to insulin acceptance and dose optimization [16-19].

The importance of complementing RCT data with real-world data, both to fill the gaps in RCT comparative effectiveness data and to ensure that data derived from RCTs are generalizable to different real-world treatment settings and patient populations, is increasingly being recognized [20]. A number of real-world studies from the US have demonstrated comparable to improved glycemic control and comparable to reduced hypoglycemia with Gla-300 vs. (mainly) first-generation BIAs in insulin- 
experienced [21-24] and insulin-naïve [23, 25] patients. However, there is a paucity of realworld comparative effectiveness research in European patient populations [26]. Non-comparative studies conducted in Europe have shown that switching to [27-29] or initiating [30] Gla-300 improves glycemic control, but the impact on hypoglycemia is unclear.

The main objectives of the current study were to compare patient demographics, clinical characteristics, glycemic control, and hypoglycemic events of T2D patients prescribed Gla300 or Gla-100 using data from clinical practice in France, Spain, and Germany. We also examined physicians' reasons for initiating Gla-300 or Gla-100.

\section{METHODS}

\section{Study Design}

This was a retrospective chart review analysis. Kantar obtained approval of the study protocol and case report form from Die Freiburger EthikKommission International (FEKI) in Germany and Sterling IRB in the US. Informed consent was obtained from all respondents before enrollment. Participating physicians were compensated at the completion of the questionnaire and three or four eCRFs. The focus of this article is on the analyses of patients switching BIA therapy to either Gla-300 or Gla-100. Additional analyses are presented in the supplement for insulin-naïve patients who were initiating either Gla-300 or Gla-100. Figure 1 shows a schematic of the study design.

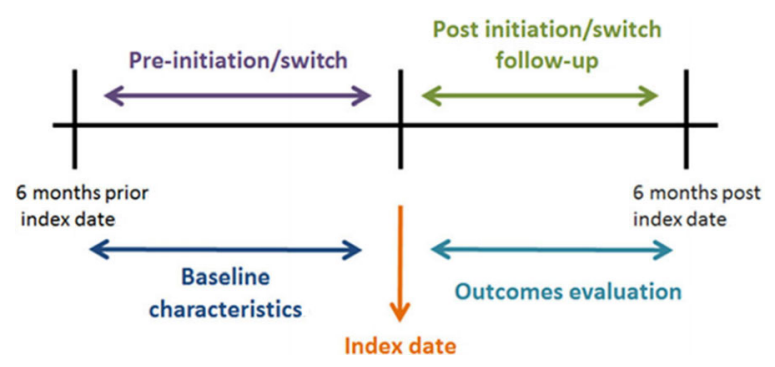

Fig. 1 Schematic of the study design

\section{The All Global Online Panel}

Physicians were recruited via the All Global online panel of physicians and healthcare professionals (https://www.allglobalcircle.com/). In the European Union, panelists are recruited from proven medical list sources. A stringent sampling procedure ensures a representative demographic cross section that takes into consideration population density by: country, specialty, region, rural vs. urban, hospital vs. office, grades, gender, and therapy/disease areas.

\section{Study Respondents}

Potential physician respondents for the present study were invited to participate in a research study. The study details were not shown in the invitation, but rather were first viewed as part of the subsequent informed consent after agreement to participate. This decreased the likelihood of bias due to self-selection for participation.

A 'blinded' approach to physician/patient de-identification was adopted; i.e., no personally identifiable information pertinent to physicians or patients was collected in this study. Demographic data, such as country, region, gender, and hospital vs. office setting, were collected and monitored to ensure that the respondents were representative of the physician population for each country.

Eligible physicians were specialized in diabetology/endocrinology, internal medicine, or general/family practice, with 3-35 years of clinical practice and were working in a clinical setting for $\geq 60 \%$ of their working hours. Each physician had treated $\geq 40$ patients with T2D monthly and had initiated $\geq 2$ patients with Gla-300 or Gla-100 in the past 12 months.

Patient Record Selection and Data Extraction Data were obtained from medical chart records, extracted by participating physicians, and from self-reported information from the physicians about their practice and attitudes toward treating patients with T2D. Patient characteristics and demographics analyzed included age, gender, country, race/ethnicity, body mass index (BMI), prior comorbidities, and Charlson Comorbidity Index (CCI). Diabetes-related characteristics included glycated hemoglobin 
$A_{1 c}(A 1 C)$, prior medication use, and hospitalization during the past 6 months. Patient chart records were selected using a random decision rule. Physicians used a randomly assigned letter of the alphabet to select a patient surname whose chart was to be included (or the next letter of the alphabet, if necessary). Each physician included 3 or 4 patient charts, with $\leq 2$ charts for each patient population (switchers to Gla-300, switchers to Gla-100, insulinnaïve initiating Gla-300, insulin-naïve initiating Gla-100).

\section{Included Patients}

Included patients were aged $\geq 18$ years at the date of insulin switching/initiation, with a diagnosis of T2D, and had switched/initiated within the previous 12 months from the date of chart selection. They were treated, under the physician's care, with Gla-300 or Gla-100 for $\geq 6$ months post-switch/initiation, had $\geq 1$ A1C laboratory value within the 6 months prior to switching/initiation, and $\geq 1 \mathrm{~A} 1 \mathrm{C}$ laboratory value 3-6 months afterwards. In the switcher population, patients had been using BIA therapy for $\geq 6$ months prior to switching. Although unlikely for patients switched to Gla100 , prior users of Gla- 300 were excluded. In the insulin-naïve patient population, patients had not previously been treated with any type of insulin (i.e., basal insulin, bolus/mealtime insulin, or pre-mix insulin).

\section{Outcome Measures}

Outcome measures included changes from baseline to 6-month follow-up in: A1C (most recent result 3-6 months after switch/initiation); incidence of total hypoglycemia, including non-severe hypoglycemia (captured in the patient chart, having been reported by phone or during an office visit) and severe hypoglycemia (requiring active assistance, such as the assistance of another person to actively administer carbohydrate, glucagon, or other resuscitative or medical interventions such as emergency or ambulance calls, or physician intervention); incidence of severe hypoglycemia; total and severe hypoglycemic events; and daily weight- adjusted insulin dose. Also, reasons were given for switching treatment or new treatment choice.

\section{Statistical Analyses}

Descriptive statistics [means and standard deviations (SDs) for continuous variables and frequencies and percentages for categorical variables] were calculated for the study variables (SAS 9.3). Bivariate analyses were used to compare baseline and post-switch/initiation characteristics of patients prescribed Gla-300 vs. Gla100 via two independent sample $t$ tests for continuous measures and $\chi^{2}$ tests for categorical variables. Generalized linear models were used to compare post-switch/initiation outcomes between patients who were prescribed Gla-300 vs. Gla-100, while adjusting for baseline differences between groups. Covariates included all variables significantly different between groups as identified in the bivariate analyses as well as variables of a-priori interest.

\section{RESULTS}

In total, 665 medical practitioners were recruited to the study during January to May 2018: 225 (33.8\%) from Spain, 211 (31.7\%) from France, and 229 (34.4\%) from Germany. The majority were general/family practice physicians $(n=322 ; 48.4 \%)$, followed by diabetologists $\quad(n=158 ; \quad 23.8 \%), \quad$ endocrinologists $(n=104 ; 15.6 \%)$ and internal medicine specialists $(n=81 ; 12.2 \%)$.

\section{Baseline Characteristics}

A total of 1108 patients who switched insulin were included, 679 to Gla-300 and 429 to Gla100. Furthermore, 1430 insulin-naïve patients who had initiated insulin were included, of whom 719 had initiated Gla-300 and 711 had initiated Gla-100.

At baseline, switchers to Gla-300 vs. switchers to Gla-100 had a higher mean BMI $(29.6 \mathrm{~kg} /$ $\mathrm{m}^{2}$ vs. $\left.28.5 \mathrm{~kg} / \mathrm{m}^{2} ; p<0.001\right)$ and lower mean A1C $(8.42 \%$ vs. $8.57 \% ; p=0.045)$ (Table 1$)$. 
Table 1 Switcher group patient disposition

\begin{tabular}{|c|c|c|c|}
\hline Characteristic & $\begin{array}{l}\text { Switched to Gla-300 } \\
(n=679)\end{array}$ & $\begin{array}{l}\text { Switched to Gla-100 } \\
(n=429)\end{array}$ & $p$ value \\
\hline Age, years & $63.1 \pm 10.6$ & $62.6 \pm 11.4$ & 0.522 \\
\hline Male & $401(59.1)$ & $241(56.2)$ & 0.344 \\
\hline Country & & & 0.074 \\
\hline Germany & $225(33.1)$ & $166(38.7)$ & \\
\hline Spain & $231(34.0)$ & $147(34.3)$ & \\
\hline France & $223(32.8)$ & $116(27.0)$ & \\
\hline Race/ethnicity & & & 0.262 \\
\hline European & $602(88.7)$ & $375(87.4)$ & \\
\hline North African & $33(4.9)$ & $19(4.4)$ & \\
\hline Other/mixed/declined to answer & $44(6.5)$ & $35(8.2)$ & \\
\hline $\mathrm{CCI}^{\mathrm{a}}$ & $0.8 \pm 1.3$ & $0.9 \pm 1.4$ & 0.738 \\
\hline BMI prior to switch, $\mathrm{kg} / \mathrm{m}^{2}$ & $29.6 \pm 5.1(n=558)$ & $28.5 \pm 3.6(n=327)$ & $<0.001$ \\
\hline $\mathrm{AlC}, \%$ & $8.42 \pm 1.15$ & $8.57 \pm 1.30$ & 0.045 \\
\hline Any hypoglycemia & $231 / 614(37.6)$ & $98 / 379(25.9)$ & $<0.001$ \\
\hline Events per patient in 6 months & $1.33 \pm 3.63(n=595)$ & $0.72 \pm 1.97(n=367)$ & 0.003 \\
\hline Severe hypoglycemia & $75 / 570(13.2)$ & $32 / 359(8.9)$ & 0.048 \\
\hline Events per patient in 6 months & $0.18 \pm 0.52(n=570)$ & $0.11 \pm 0.36(n=359)$ & 0.026 \\
\hline Total insulin dose prior to switch, $\mathrm{U} / \mathrm{kg} /$ day & $0.40 \pm 0.22(n=574)$ & $0.38 \pm 0.23(n=338)$ & 0.107 \\
\hline Baseline BIA prior to switch & & & $<0.001$ \\
\hline Gla-100 & $281(41.4)$ & $0(0)$ & \\
\hline Insulin detemir & $194(28.6)$ & $203(47.3)$ & \\
\hline NPH insulin & $153(22.5)$ & $171(39.9)$ & \\
\hline Abasaglar & $22(3.2)$ & $25(5.8)$ & \\
\hline Insulin degludec & $15(2.2)$ & $9(2.1)$ & \\
\hline Other/unavailable/unknown & $14(2.1)$ & $21(4.9)$ & \\
\hline \multicolumn{4}{|l|}{ Prior comorbidities } \\
\hline Hypertension & $480(70.7)$ & $289(67.4)$ & 0.242 \\
\hline Obesity/morbid obesity & $147(21.6)$ & $52(12.1)$ & $<0.001$ \\
\hline Depression & $97(14.3)$ & $50(11.7)$ & 0.209 \\
\hline Congestive heart failure & $78(11.5)$ & $52(12.1)$ & 0.750 \\
\hline T2D with chronic complication & $76(11.2)$ & $36(8.4)$ & 0.132 \\
\hline Renal disease & $64(9.4)$ & $26(6.1)$ & 0.046 \\
\hline
\end{tabular}


Table 1 continued

\begin{tabular}{llll}
\hline Characteristic & $\begin{array}{l}\text { Switched to Gla-300 } \\
(\boldsymbol{n}=\mathbf{6 7 9 )}\end{array}$ & $\begin{array}{l}\text { Switched to Gla-100 } \\
(\boldsymbol{n}=\mathbf{4 2 9})\end{array}$ & $\boldsymbol{p}$ value \\
\hline Myocardial infarction & $60(8.8)$ & $49(11.4)$ & 0.159 \\
Nephropathy due to diabetes & $47(6.9)$ & $14(3.3)$ & 0.009 \\
TIA/stroke & $41(6.0)$ & $12(2.8)$ & 0.014 \\
Foot or leg ulcer & $17(2.5)$ & $7(1.6)$ & 0.331 \\
Prior diabetes medications & & & 0.642 \\
Metformin & $310(45.7)$ & $202(47.1)$ & 0.953 \\
DPP-4 inhibitor & $99(14.6)$ & $62(14.5)$ & 0.207 \\
Sulfonylurea & $56(8.2)$ & $45(10.5)$ & 0.454 \\
GLP-1RA & $21(3.1)$ & $10(2.3)$ & 0.431 \\
SGLT-2 inhibitor & $14(2.1)$ & $12(2.8)$ & \\
Fixed combinations & & & 0.354 \\
BIA + GLP-1RA & $124(18.3)$ & $88(20.5)$ & 0.002 \\
DPP-4 inhibitor + metformin & $83(12.2)$ & $28(6.5)$ & 0.920 \\
SGLT-2 inhibitor + metformin & $10(1.5)$ & $6(1.4)$ & $5(1.2)$ \\
Sulfonylurea + metformin & $9(1.3)$ & $1(0.2)$ & 0.816 \\
SGLT-2 inhibitor + DPP-4 inhibitor & $1(0.1)$ & $1.6 \pm 5.2(n=380)$ & 0.743 \\
Hospitalized in past 6 months & $1.0 \pm 1.7(n=621)$ & 0.011 \\
\hline Da & &
\end{tabular}

Data are expressed as mean $\pm \mathrm{SD}, n(\%)$, or $n / N(\%)$ in case of missing data

$A I C$ hemoglobin Alc, BIA basal insulin analog, BMI body mass index, CCI Charlson Comorbidity Index, DPP-4 dipeptidyl peptidase-4, Gla-100 insulin glargine $100 \mathrm{U} / \mathrm{ml}$, Gla-300 insulin glargine $300 \mathrm{U} / \mathrm{ml}$, GLP-1RA glucagon-like peptide 1 receptor agonist, $N P H$ neutral protamine Hagedorn, $S D$ standard deviation, $T I A$ transient ischemic attack, $S G L T 2$ sodium-glucose transport protein 2, T2D type 2 diabetes

${ }^{a}$ Excluding diabetes and diabetes with complications

Patients who switched to Gla-300 had most commonly previously been treated with Gla$100(41.4 \%)$ or insulin detemir (28.6\%), and $83.9 \%$ were using bolus/meal-time insulin. The majority of patients switching to Gla-100 had previously been taking insulin detemir $(47.3 \%)$ or neutral protamine Hagedorn (NPH) insulin $(39.9 \%)$ (Table 1). Prior to switching, the incidence $(34.0 \%$ vs. $22.8 \% ; p<0.001)$ and mean rate (1.33 vs. 0.72 events per patient in 6 months; $p=0.003$ ) of hypoglycemia were higher in patients who switched to Gla-300 vs. Gla-100 (Table 1).
The following comorbidities were more common in patients switching to Gla-300 vs. Gla-100: obesity/morbid obesity $(21.6 \%$ vs. $12.1 \% ; p<0.001)$, renal disease $(9.4 \%$ vs. $6.1 \%$; $p=0.046)$, nephropathy due to diabetes $(6.9 \%$ vs. $3.3 \% ; p=0.009)$, and transient ischemic attack/stroke $(6.0 \%$ vs. $2.8 \% ; p=0.014)$.

Patient demographics and comorbidities for the insulin-naïve cohort are shown in Table S1 in the supplementary material. Insulin-naïve patients tended to have higher baseline A1C, less baseline hypoglycemia, higher use of oral diabetes medications, lower CCI, and fewer prior hospitalizations than the switcher group. 

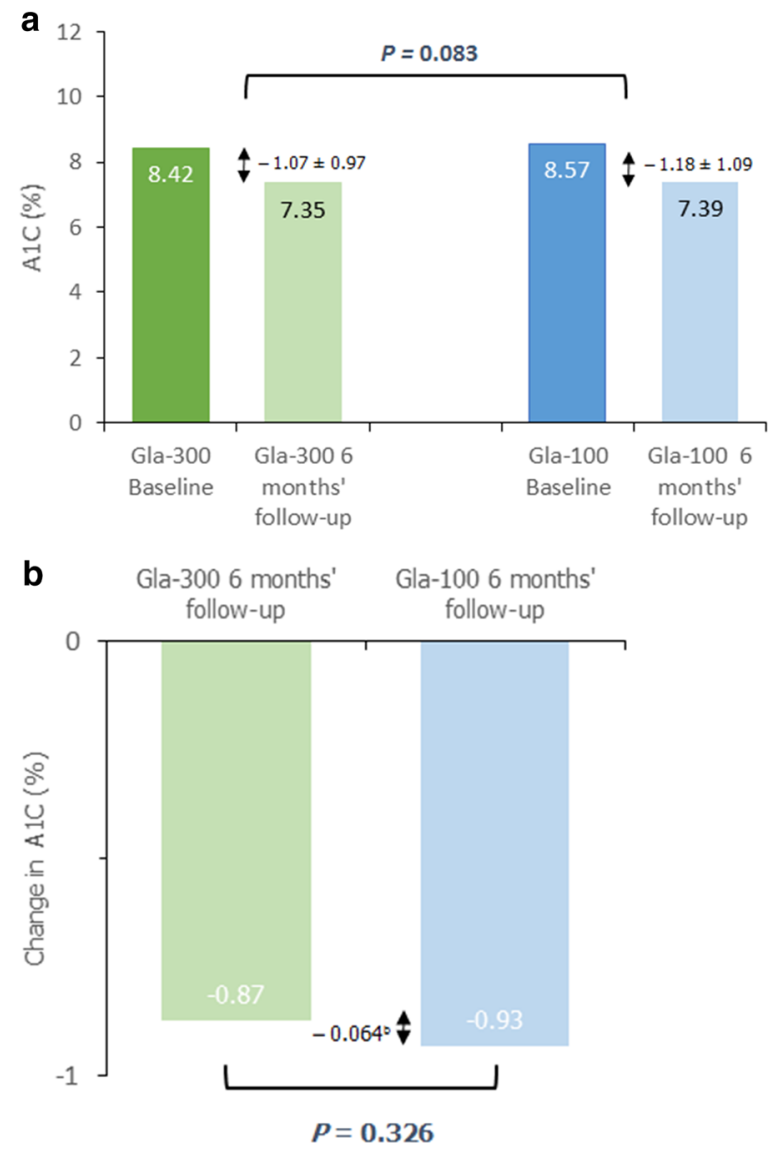

Fig. 2 Switcher group: a unadjusted and b adjusted ${ }^{a}$ change in A1C from baseline to follow-up. adjusted for pre/post A1C difference, age, gender, baseline TIA/stroke, obesity, renal disease, nephropathy due to diabetes, metformin, DPP-4 inhibitor, sulfonylurea, bolus/mealtime insulin use, prior NPH insulin, and other basal insulin use, prior hospitalization, missing hospitalization data, country, ethnicity, insurance, treatment expectation, prior hypoglycemia, and time from treatment initiation to

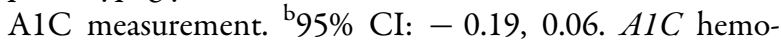
globin A1c, CI confidence interval, DPP-4 dipeptidyl peptidase-4, Gla-100 insulin glargine $100 \mathrm{U} / \mathrm{ml}$, Gla-300 insulin glargine $300 \mathrm{U} / \mathrm{ml}, N P H$ neutral protamine Hagedorn, TIA transient ischemic attack

\section{Changes in A1C and Hypoglycemia}

At 3-6-month follow-up, mean reductions in A1C from baseline were similar for insulin-experienced patients switching to Gla-300 vs. Gla100 (Fig. 2a). When adjusted for baseline characteristics, the reductions remained

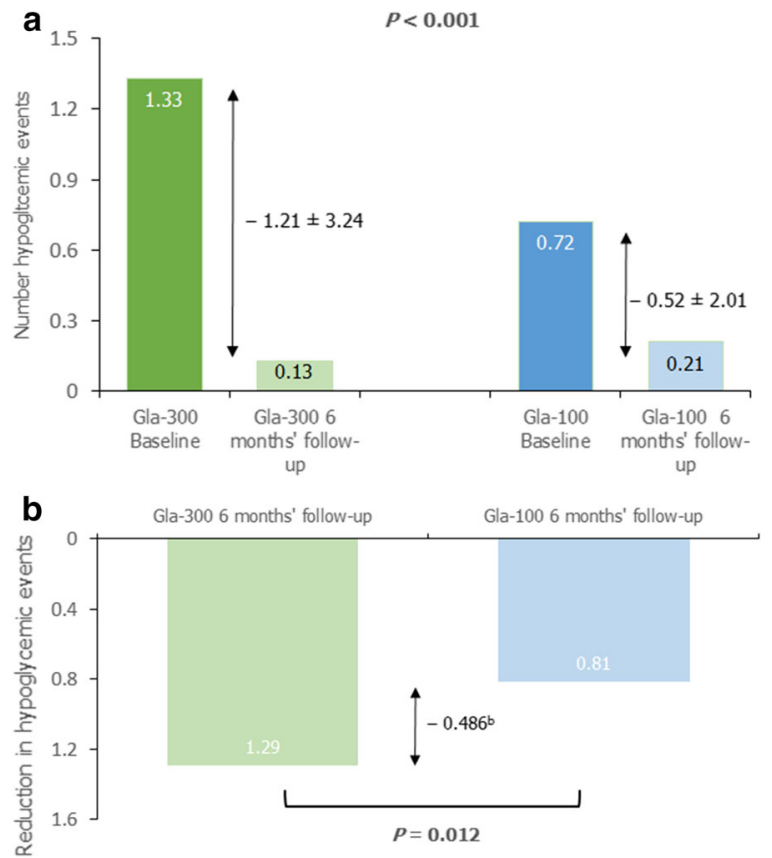

Fig. 3 Switcher group reduction in number of hypoglycemic events: a unadjusted data and $\mathbf{b}$ adjusted data ${ }^{a}$. ${ }^{a}$ Adjusted for pre/post hypoglycemia, age, gender, baseline TIA/stroke, obesity, renal disease, nephropathy due to diabetes, metformin, DPP-4 inhibitor, sulfonylurea, bolus/ meal-time insulin use, prior NPH insulin and other basal insulin use, prior hospitalization, missing hospitalization data, country, ethnicity, insurance, treatment expectation, difference in post-pre A1C (or difference in A1C) $(n=927) .{ }^{\text {b}} 95 \% \mathrm{CI}:-0.86,-0.11$. A1C hemoglobin Alc, $C I$ confidence interval, $D P P-4$ dipeptidyl peptidase-4, Gla-100 insulin glargine $100 \mathrm{U} / \mathrm{ml}$, Gla-300 insulin glargine $300 \mathrm{U} / \mathrm{ml}, \mathrm{NPH}$ neutral protamine Hagedorn, TIA transient ischemic attack

comparable: $-0.87 \%$ vs. $-0.93 \%$, respectively; $p=0.326$; Fig. 2b).

The mean reduction in hypoglycemic events per patient over 6 months was greater for Gla300 switchers vs. Gla-100 switchers when unadjusted (Fig. 3a) and when adjusted for baseline characteristics (-1.29 vs. -0.81 , respectively; $p=0.012$; Fig. $3 b$ ). So too was the adjusted reduction in severe hypoglycemic events $\quad(-0.17 \quad$ vs. $-0.10, \quad$ respectively; $p=0.038)$.

In the insulin-naïve group, Gla-300 vs. Gla100 initiators had a significantly smaller adjusted reduction in $\mathrm{A} 1 \mathrm{C}(-1.21$ vs. - 1.35; 


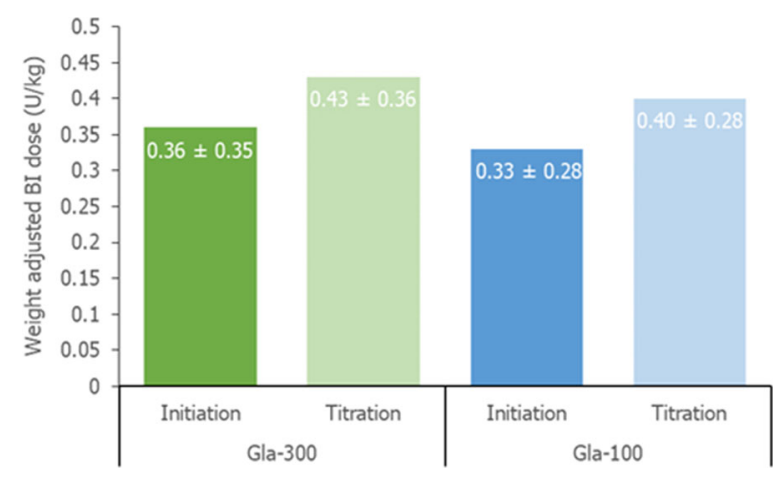

Fig. 4 Switcher group weight-adjusted insulin dose of Gla300 and Gla-100a. BIA basal insulin analog, Gla-100 insulin glargine $100 \mathrm{U} / \mathrm{ml}$, Gla-300 insulin glargine 300 $\mathrm{U} / \mathrm{ml}$

$p=0.014 ;$ Fig. S1 in the supplementary material) and a significantly greater adjusted reduction in hypoglycemia $(-0.20$ vs. +0.04 ; $p=0.001$; Fig. S2 in the supplementary material).

\section{Change in Dose}

In the insulin-experienced group, the mean \pm SD number of weeks of titration following insulin switch were similar for Gla-300 and Gla100: $5.7 \pm 4.0$ and $5.6 \pm 3.6$ weeks, respectively; $p=0.751$. There was no significant difference in weight-adjusted BIA dose between the two groups at initiation or during the titration phase (Fig. 4).

Weight-adjusted insulin doses in the insulinnaïve groups are shown in Fig. S3 in the supplementary material.

\section{Changes in Other Diabetes Medications}

Use of bolus/meal-time insulin decreased considerably after switching to Gla-300 or Gla-100, but use of other diabetes medications was relatively stable (Table 1 vs. Table S2 in the supplementary material). Among insulin-naïve patients, the use of dipeptidyl peptidase-4 inhibitors, sulfonylureas, and fixed-combination dipeptidyl peptidase- 4 inhibitor + metformin decreased considerably, but use of other diabetes medications was relatively stable (Tables S1 vs. S3 in the supplementary material).

\section{Reasons for BIA Choice}

The reasons for treatment choice are shown in Fig. 5a. Reasons that were significantly more often cited for Gla-300 vs. Gla-100 included a lower risk of hypoglycemia (36.7\% vs. $25.6 \%$; $p<0.001)$ and clinical benefits other than glycemic control $(41.1 \%$ vs. $33.8 \% ; p=0.015)$. Reasons that were significantly more often cited for Gla-100 vs. Gla-300 were: familiarity (31.7\% vs. $24.7 \% ; p=0.011)$, treatment guidelines $(10.5 \%$ vs. $7.1 \% ; p=0.046)$, and formulary reasons $(8.9 \%$ vs. $5.2 \% ; p=0.016)$. Comorbidities/contraindications did not appear to be a driver of treatment decisions (Fig. 5a).

The split between the main treatment expectations differed significantly between Gla300 and Gla-100 (Fig. 5b). While achievement of a better glycemic target was the most common expectation for Gla-300 and Gla-100 (49.9\% and $57.6 \%$, respectively), the second most common treatment expectation was a lower risk of hypoglycemia for Gla-300 (23.3\%) but being easier to titrate for Gla-100 (20.7\%). In insulin-naïve patients, results were broadly similar (Fig. S4 in the supplementary material).

\section{Outcomes by Country}

In bivariate comparisons, insulin-experienced patients in Spain, Germany, and France had similar $>1 \%$ A1C reductions after switching treatments (Table S4 in the supplementary material). The reductions in hypoglycemic events were significantly different between patients switched to Gla-300 vs. Gla-100 in Spain $(-1.78$ vs. $-0.82 ; p=0.019)$ and France $(-1.11$ vs. $-0.52 ; p=0.048)$, but did not reach statistical significance in Germany $(-0.72$ vs. $-0.26 ; p=0.078$ ) (Table S4 in the supplementary material).

In insulin-naïve patients, results by country were broadly similar to the main analysis (Table S5 in the supplementary material). 


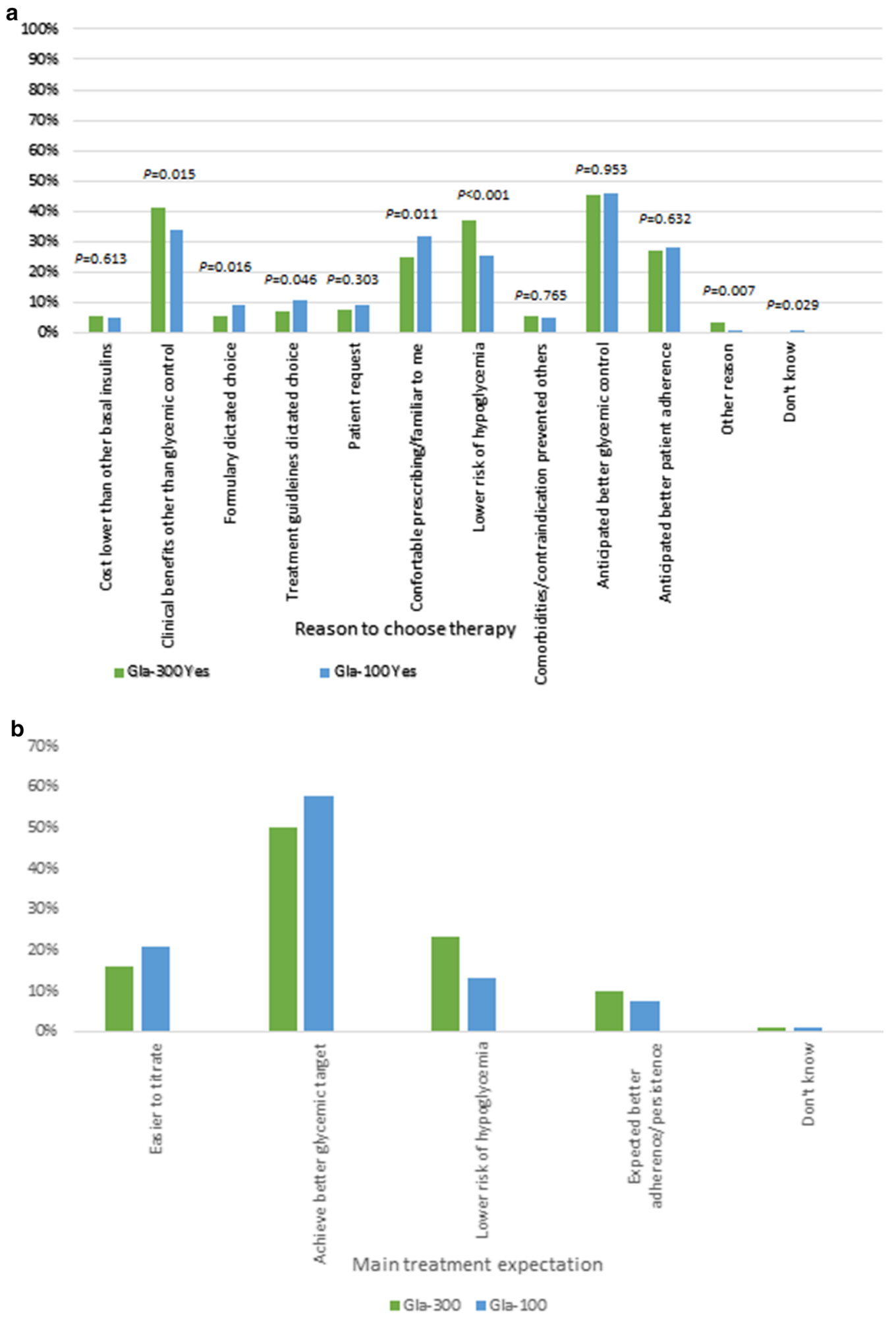

Fig. 5 Switcher group: a physicians' reasons for treatment decisions and $\mathbf{b}$ physician's main treatment expectations ${ }^{\mathrm{a}}$. ${ }^{a}$ Overall Gla-300 vs. Gla-100: $p<0.001$. Gla-100 insulin glargine $100 \mathrm{U} / \mathrm{ml}$, Gla-300 insulin glargine $300 \mathrm{U} / \mathrm{ml}$ 


\section{DISCUSSION}

In this comparative real-world analysis of European data from insulin-experienced patients who switched BIA therapy to either Gla-300 or Gla-100, at 6 months after switching, improvements in glycemic control were similar in both treatment groups, with $\mathrm{A} 1 \mathrm{C}$ reductions of about $1 \%$. In multivariate analyses adjusting for baseline differences, switching to Gla-300 vs. Gla-100 was associated with a significantly greater reduction in the number of hypoglycemic events. Results for insulin-naïve patients initiating Gla-300 or Gla-100 were broadly similar, with A1C reductions of about $1.2-1.3 \%$ from baseline, and a significant difference in adjusted hypoglycemic events, with Gla-300 showing a decrease while Gla-100 showed a slight increase. Both groups may have benefited from further titration in terms of their A1C improvement.

At baseline, patients switching BIA therapy to Gla-300 vs. Gla-100 had a higher BMI, higher incidence of hypoglycemia, lower $\mathrm{A} 1 \mathrm{C}$, and a greater burden of comorbidities, while those initiating Gla-300 vs. Gla-100 were younger, were more often male and of European race/ ethnicity, had a higher BMI, had more hypoglycemia, and had more comorbidities. In a previous study conducted in Germany and Austria, patients with T2D who were prescribed Gla-300 (switch or initiate) were also younger, had a higher BMI, and had lower A1C [31]. Our outcomes data are generally in line with those from the EDITION series of RCTs comparing Gla-300 and Gla-100. In the EDITION T2D studies, Gla-300 and Gla-100 were found to achieve similar glycemic control, but Gla-300 was associated with improvements in some hypoglycemia end points in insulin-experienced patients treated with basal-bolus therapy $[5]$ or oral + basal therapy $[6,7]$ and in insulinnaïve patients $[8,9]$.

In the EDITION 1 study, which recruited patients on basal and meal-time insulin, switching to Gla-300 resulted in a similar reduction in A1C after 6 months $(-0.83 \%)$ [5] as that seen in our study $(-0.87 \%$, adjusted). This result is notable given the assumed advantages of the RCT setting for achieving therapeutic end points, including treat-to-target titration schemes and the increased attention provided by the structure of the RCT, which may improve patient adherence to therapy [32]. However, it should be noted that mean baseline A1C was lower in EDITION 1 than in the present study ( $8.16 \%$ vs. $8.47 \%$, respectively), body weight was higher (106 vs. $84 \mathrm{~kg}$ ), and there was a requirement for a relatively high baseline use of basal insulin ( $\geq 42 \mathrm{U} /$ day) in EDITION 1 [5], all factors that could potentially have an impact on observed $\mathrm{A} 1 \mathrm{C}$ changes.

In the EDITION 3 study in insulin-naïve patients [8], mean baseline A1C was also lower than in the insulin-naive population in the present study $(8.54 \%$ vs. $8.68 \%)$; however, reductions in $\mathrm{A} 1 \mathrm{C}$ were slightly more comparable (approximately $-1.5 \%$ vs. $-1.31 \%$ in the present study). In the current study, the small but significantly greater A1C reduction among Gla-100 vs. Gla-300 initiators $(-0.14 \%$, $p=0.014)$ is unlikely to be of clinical significance. This was also at the cost of a slight increase in hypoglycemia, possibly the result of the different attitude toward patient management (i.e., greater focus on better glycemic control than on reduced hypoglycemia).

Our data also add to a growing body of realworld evidence demonstrating the effectiveness of switching to Gla-300. In previous real-world studies, insulin-experienced patients who switched to Gla-300 consistently showed important reductions in A1C [18, 21-23, 27-29, 32]. In those studies with a (mainly) first-generation BIA propensity score-matched comparator group, Gla-300 showed similar improvements in glycemic control [21-23]. None of these comparative studies were conducted in Europe, but the results of two pragmatic studies that were mainly conducted in Europe have recently been published [26]. These were randomized real-world insulin-experienced and -naïve patients to Gla-300 or standard-of-care BIA (mainly Gla-100) [26]. Gla-300 was non-inferior for A1C reduction among insulin-naïve but not insulin-experienced patients [26].

In the current study, Gla-300 switchers had a larger unadjusted reduction in hypoglycemia events than Gla-100 switchers, but this was 
mainly due to a higher number of events during baseline as the number during follow-up was similar in both groups. However, after controlling for covariates, the reduction in hypoglycemia events with Gla-300 remained significant. Here, once again, our data are in line with other real-world studies, which have shown reduced hypoglycemia risk with Gla-300 vs. (mainly) first-generation BIAs $[18,21-23,33]$. In the above-mentioned pragmatic studies that were mainly conducted in Europe, most hypoglycemia end points were similar for Gla-300 and standard-of-care BIA, but there were some significant reductions in nocturnal hypoglycemia with Gla-300 [26]. These hypoglycemia advantages are presumed to be derived from the more stable PK/PD profile of Gla-300 [13-15].

The higher rate of baseline hypoglycemia in the Gla-300 switcher group is unsurprising, given that physicians cited a lower risk of hypoglycemia as a major reason for switching to Gla-300 and a lower rate of hypoglycemia was more often a primary expectation after switching in the patients for whom Gla-300 was chosen. These data are in line with a US study, where hypoglycemia advantages were stated as a reason to switch to Gla-300 [18]. However, a study from The Netherlands reported that reduced volume, improved flexibility, and better quality of life were more often cited as reasons for switching to Gla-300 than fear of hypoglycemia [19]. Interestingly, in a study that included patients with diabetes from 17 countries, the proportions who were concerned about hypoglycemia varied widely by country, from approximately 30\% (in The Netherlands) to around $80 \%$ (in Algeria) [4]. In the current study, the higher rates of comorbidities in the Gla-300 group did not appear to be a driver of treatment choices, with only approximately 5\% of physicians stating that this was a reason for switching, a similar proportion to that seen in the Gla-100 group. Familiarity with therapy was also previously seen to be a more frequent reason given by physicians when prescribing Gla100 [18], and this was also observed in the present study. Gla-300 had relatively recently been approved (2015 in Germany and 2017 in France and Spain) when participating physicians were recruited to the study (early 2018), and so their reasoning for treatment selection might be expected to change as they gain experience and familiarity over time. Responses for the insulin-naïve group suggest that drivers of physician treatment choice were similar to those reported for the insulin-experienced group who were switching.

There appeared to be some country-specific differences in rates of hypoglycemia following insulin switching in our study, with a difference in severe hypoglycemia in favor of Gla-300 evident in Spain alone. However, it should be noted that the country-specific subgroup analyses involved relatively small sample sizes. Additionally, interpretation of results is complicated by differences in the approach to insulin titration that may be evident in different countries. Other studies have reported differences in hypoglycemia between different European countries, although these were more evident prior to insulin initiation than afterwards [34]. Such differences may, in part, be due to country-specific differences in factors such as the way that hypoglycemia is defined or recorded, patient characteristics, or the way that BIAs are titrated. Large global observational studies such as DISCOVER $(n=15,000)$, which is investigating real-world treatment patterns across 38 countries, may help shed light on the reasons behind such international differences in outcomes [35]. The REALI project, a pooled analysis of European studies, may also provide further information on Gla-300 use in Europe [36].

Our study has a number of limitations. As a retrospective analysis of patient medical records sourced from self-selected physicians, the data here represent a convenience sample that may not be generalizable to all patients using Gla300 or Gla-100 in the countries included. Given the time frame of the study and the introduction of Gla-300 to the market, more patients were perhaps being switched to Gla-300 than other BIAs. Therefore, it was relatively difficult to find eligible patients who had recently switched to Gla-100. Although the analysis plan required similar numbers of patients in both the switcher and naïve groups, despite extending the 'recruitment' period, fewer of these patients 
were included in the database relative to the other groups. The analysis was nevertheless undertaken as sufficient numbers were available.

The data submitted by physicians may be subject to channeling bias and unmeasured confounders, such as socioeconomic status, or disease severity, and country-specific differences in how hypoglycemia is reported and how insulin titration is managed. Indeed, patients in the two groups do show some differences, and physicians were known to initiate therapies for different reasons. Analyses adjusted for baseline differences should minimize their influence, but some element of confounding may remain. Propensity score matching of included patients was considered but rejected in favor of the regression method because of the reductive nature of the method. Bolus/meal-time insulin use among BIA switchers and non-insulin diabetes medication use among BIA initiators fell dramatically after switching/initiation of Gla300 or Gla-100, which could have impacted A1C and hypoglycemia results. However, as the changes were similar in both BIA groups, this should not have overly affected the betweengroup comparisons. Lastly, hypoglycemia levels were likely to be underestimated because of a reliance upon recorded events that were significant enough to show up in medical charts.

Despite the limitations, there are significant strengths of this study. Medical record abstraction provides an opportunity to assess realworld data outside the highly controlled setting of clinical trials. Data were collected from relatively large samples from three European countries, healthcare professional profiles were diverse, and it was possible to assess clinical, biologic, and BIA dose data, even if based on chart reviews. The random decision rule design may have helped control for some confounding aspects of the convenience sample, such as a tendency for physicians to select the most recent charts available.

\section{CONCLUSION}

This comparative European real-world studywhich examined patients who were switched to or initiated Gla-300 or Gla-100-supports the results from RCTs and US real-world comparative studies that Gla-300 provides comparable glycemic control to Gla-100, but with a reduced risk of hypoglycemia. This study provides important additional evidence in European patients, as there were previously little comparative data in this population. Regarding choice of therapy, physicians were more likely to select Gla-300 to achieve a lower risk of hypoglycemia, whereas familiarity was more important when selecting Gla-100. It would be interesting to repeat this analysis when physicians are equally familiar with the use of Gla300 and Gla-100.

\section{ACKNOWLEDGEMENTS}

Funding. This study, the Rapid Service and Open Access Fees were funded by Sanofi US, Inc. All authors had full access to all of the data in this study and take complete responsibility for the integrity of the data and accuracy of the data analysis.

Medical Writing Assistance. The authors received writing/editorial support in the preparation of this manuscript provided by Joseph Worrall, PhD, of Excerpta Medica, funded by Sanofi US, Inc.

Authorship. All named authors meet the International Committee of Medical Journal Editors (ICMJE) criteria for authorship of this article, take responsibility for the integrity of the work as a whole, and have given final approval for the version to be published.

Disclosures. Javier Escalada receives honoraria for advisory boards and speakers bureaus from Eli Lilly, MSD, Novo Nordisk, Sanofi, AstraZeneca, and Boehringer Ingelheim. Fabrice Bonnet receives honoraria for advisory boards from Amgen, AstraZeneca, Boehringer Ingelheim, Lilly, MSD, Novo Nordisk and Sanofi. Jasmanda Wu, Mireille Bonnemaire and Charlie Nicholls are employees of Sanofi and may own stock/shares in the company; Shaloo Gupta and 
M. Janelle Cambron-Mellott are employees of Kantar, who received funding from Sanofi to conduct this study. Dirk Müller-Wieland receives honoraria for advisory boards and speaker bureaus from Amgen, AstraZeneca, Boehringer Ingelheim GmbH, NovoNordisk, MSD, Novartis, and Sanofi.

Compliance with Ethics Guidelines. Kantar obtained approval of the study protocol and case report form from Die Freiburger EthikKommission International (FEKI) and Sterling IRB in the US. Informed consent was obtained from all respondents before enrollment. Participating physicians were compensated at the completion of the questionnaire and three or four eCRFs.

Data Availability. Qualified researchers may request access to patient-level data and related study documents including the clinical study report, study protocol with any amendments, blank case report form, statistical analysis plan, and dataset specifications. Patient level data will be anonymized and study documents will be redacted to protect the privacy of trial participants. Further details on Sanofi's data sharing criteria, eligible studies, and process for requesting access can be found at: https://www.clinicalstudydatarequest.com.

Open Access. This article is licensed under a Creative Commons Attribution-NonCommercial 4.0 International License, which permits any non-commercial use, sharing, adaptation, distribution and reproduction in any medium or format, as long as you give appropriate credit to the original author(s) and the source, provide a link to the Creative Commons licence, and indicate if changes were made. The images or other third party material in this article are included in the article's Creative Commons licence, unless indicated otherwise in a credit line to the material. If material is not included in the article's Creative Commons licence and your intended use is not permitted by statutory regulation or exceeds the permitted use, you will need to obtain permission directly from the copyright holder. To view a copy of this licence, visit http://creativecommons.org/licenses/by$\mathrm{nc} / 4.0 /$.

\section{REFERENCES}

1. World Health Organization Regional Office for Europe. The challenge of diabetes. https://www. euro.who.int/en/health-topics/noncommunicablediseases/diabetes/data-and-statistics. Accessed May 2020.

2. Ceriello A, deValk HW, Guerci B, et al. The burden of type 2 diabetes in Europe: current and future aspects of insulin treatment from patient and healthcare spending perspectives. Diabetes Res Clin Pract. 2020;161:108053.

3. Davies MJ, D'Alessio DA, Fradkin J, Management of Hyperglycemia in Type 2 Diabetes, et al. A consensus report by the American Diabetes Association (ADA) and the European Association for the Study of Diabetes (EASD). Diabetes Care. 2018;2018(41): 2669-701.

4. Nicolucci A, Kovacs Burns K, Holt RI, et al. Diabetes attitudes, wishes and needs second study (DAWN2): cross-national benchmarking of diabetes-related psychosocial outcomes for people with diabetes. Diabet Med. 2013;30:767-77.

5. Riddle MC, Bolli GB, Ziemen $\mathrm{M}$, et al. New insulin glargine 300 units/mL versus glargine 100 units $/ \mathrm{mL}$ in people with type 2 diabetes using basal and mealtime insulin: glucose control and hypoglycemia in a 6-month randomized controlled trial (EDITION 1). Diabetes Care. 2014;37:2755-62.

6. Yki-Jarvinen H, Bergenstal R, Ziemen M, et al. New insulin glargine 300 units $/ \mathrm{mL}$ versus glargine 100 units/mL in people with type 2 diabetes using oral agents and basal insulin: glucose control and hypoglycemia in a 6-month randomized controlled trial (EDITION 2). Diabetes Care. 2014;37:3235-43.

7. Terauchi Y, Koyama M, Cheng X, et al. New insulin glargine $300 \mathrm{U} / \mathrm{ml}$ versus glargine $100 \mathrm{U} / \mathrm{ml}$ in Japanese people with type 2 diabetes using basal insulin and oral antihyperglycaemic drugs: glucose control and hypoglycaemia in a randomized controlled trial (EDITION JP 2). Diabetes Obes Metab. 2016;18:366-74.

8. Bolli GB, Riddle MC, Bergenstal RM, et al. New insulin glargine $300 \mathrm{U} / \mathrm{ml}$ compared with glargine $100 \mathrm{U} / \mathrm{ml}$ in insulin-naive people with type 2 diabetes on oral glucose-lowering drugs: a randomized controlled trial (EDITION 3). Diabetes Obes Metab. 2015;17:386-94. 
9. Ji L, Kang ES, Dong X, et al. Efficacy and safety of insulin glargine $300 \mathrm{U} / \mathrm{mL}$ versus insulin glargine $100 \mathrm{U} / \mathrm{mL}$ in Asia Pacific insulin-naive people with type 2 diabetes: the EDITION AP randomized controlled trial. Diabetes Obes Metab. 2020;22:612-21.

10. Roussel R, Ritzel R, Boelle-Le Corfec E, Balkau B, Rosenstock J. Clinical perspectives from the BEGIN and EDITION programmes: trial-level meta-analyses outcomes with either degludec or glargine 300 $\mathrm{U} / \mathrm{mL}$ vs glargine $100 \mathrm{U} / \mathrm{mL}$ in T2DM. Diabetes Metab. 2018;44:402-9.

11. Ritzel R, Roussel R, Giaccari A, Vora J, BrulleWohlhueter C, Yki-Jarvinen H. Better glycaemic control and less hypoglycaemia with insulin glargine $300 \mathrm{U} / \mathrm{mL}$ vs glargine $100 \mathrm{U} / \mathrm{mL}$ : 1-year patient-level meta-analysis of the EDITION clinical studies in people with type 2 diabetes. Diabetes Obes Metab. 2018;20:541-8.

12. Ritzel R, Roussel R, Bolli GB, et al. Patient-level meta-analysis of the EDITION 1, 2 and 3 studies: glycaemic control and hypoglycaemia with new insulin glargine $300 \mathrm{U} / \mathrm{ml}$ versus glargine $100 \mathrm{U} / \mathrm{ml}$ in people with type 2 diabetes. Diabetes Obes Metab. 2015;17:859-67.

13. Becker RH, Dahmen R, Bergmann K, Lehmann A, Jax T, Heise T. New insulin glargine 300 Units $\mathrm{mL}^{-1}$ provides a more even activity profile and prolonged glycemic control at steady state compared with insulin glargine 100 Units $\mathrm{mL}^{-1}$. Diabetes Care. 2015;38:637-43.

14. Owens DR, Bailey TS, Fanelli CG, Yale JF, Bolli GB. Clinical relevance of pharmacokinetic and pharmacodynamic profiles of insulin degludec $(100,200$ $\mathrm{U} / \mathrm{mL})$ and insulin glargine $(100,300 \mathrm{U} / \mathrm{mL})-\mathrm{a}$ review of evidence and clinical interpretation. Diabetes Metab. 2019;45:330-40.

15. Clements JN, Threatt T, Ward E, Shealy KM. Clinical pharmacokinetics and pharmacodynamics of insulin glargine $300 \mathrm{U} / \mathrm{mL}$. Clin Pharmacokinet. 2017;56:449-58.

16. Peyrot M, Barnett AH, Meneghini LF, SchummDraeger PM. Factors associated with injection omission/non-adherence in the global attitudes of patients and physicians in insulin therapy study. Diabetes Obes Metab. 2012;14:1081-7.

17. Peyrot M, Barnett AH, Meneghini LF, SchummDraeger PM. Insulin adherence behaviours and barriers in the multinational global attitudes of patients and physicians in insulin therapy study. Diabet Med. 2012;29:682-9.

18. Gupta S, Wang H, Skolnik N, et al. Treatment dosing patterns and clinical outcomes for patients with type 2 diabetes starting or switching to treatment with insulin glargine (300 units per milliliter) in a real-world setting: a retrospective observational study. Adv Ther. 2018;35:43-55.

19. Wieringa TH, de Wit M, Twisk JW, Snoek FJ. Improved diabetes medication convenience and satisfaction in persons with type 2 diabetes after switching to insulin glargine $300 \mathrm{U} / \mathrm{mL}$ : results of the observational OPTIN-D study. BMJ Open Diabetes Res Care. 2018;6:e000548.

20. Sherman RE, Anderson SA, Dal Pan GJ, et al. Realworld evidence-what is it and what can it tell us? N Engl J Med. 2016;375:2293-7.

21. Bailey TS, Wu J, Zhou FL, et al. Switching to insulin glargine 300 units/mL in real-world older patients with type 2 diabetes (DELIVER 3). Diabetes Obes Metab. 2019;21:2384-93.

22. Zhou FL, Ye F, Berhanu P, et al. Real-world evidence concerning clinical and economic outcomes of switching to insulin glargine 300 units/mL vs other basal insulins in patients with type 2 diabetes using basal insulin. Diabetes Obes Metab. 2018;20: 1293-7.

23. Pettus J, Roussel R, Liz Zhou F, et al. Rates of hypoglycemia predicted in patients with type 2 diabetes on insulin glargine $300 \mathrm{U} / \mathrm{ml}$ versus firstand second-generation basal insulin analogs: the real-world LIGHTNING study. Diabetes Ther. 2019;10:617-33.

24. Zhou FL, Nicholls C, Xie L, Wang Y, Vaidya N, Meneghini LF. Hypoglycaemia and treatment patterns among insulin-treated patients with type 2 diabetes who switched to insulin glargine 300 units/mL versus other basal insulin in a real-world setting. Endocrinol Diabetes Metab. 2019;2:e00073.

25. Bailey TS, Zhou FL, Gupta RA, et al. Glycaemic goal attainment and hypoglycaemia outcomes in type 2 diabetes patients initiating insulin glargine 300 units/mL or 100 units/mL: real-world results from the DELIVER naive cohort study. Diabetes Obes Metab. 2019;21:1596-605.

26. Freemantle N, Mauricio D, Giaccari A, et al. Realworld outcomes of treatment with insulin glargine $300 \mathrm{U} / \mathrm{mL}$ versus standard-of-care in people with uncontrolled type 2 diabetes mellitus. Curr Med Res Opin. 2020;36:571-81.

27. Gourdy P, Bahloul A, Boultif Z, Gouet D, Guerci B. Efficacy and safety of switching patients inadequately controlled on basal insulin to insulin glargine $300 \mathrm{U} / \mathrm{mL}$ : the TRANSITION 2 study. Diabetes Ther. 2020;11:147-59.

28. Wolnik B, Wiza D, Szczepanik T, Syta A, Klupa T. Switching from neutral protamine hagedorn 
insulin to insulin glargine $300 \mathrm{U} / \mathrm{mL}$ improves glycaemic control and reduces hypoglycaemia risk: results of a multicentre, prospective, observational study. J Diabetes Res. 2020;2020:8751348.

29. Wiesli P, Schories M. Improved glycemic control with insulin glargine $300 \mathrm{U} / \mathrm{mL}$ (Toujeo ${ }^{\circledR}$ ) in patients with type 2 diabetes: real-world effectiveness in Switzerland. Diabetes Ther. 2018;9:2325-34.

30. Pfohl M, Jornayvaz FR, Fritsche A, et al. Effectiveness and safety of insulin glargine $300 \mathrm{U} / \mathrm{mL}$ in insulin-naive patients with type 2 diabetes after failure of oral therapy in a real-world setting. Diabetes Obes Metab. 2020;22:759-66.

31. Bohn B, Bramlage $P$, Wagner $C$, et al. Which patients from routine care use the new insulin analogue glargine U300 compared to patients with glargine U100: a multicenter analysis of 14,123 patients with insulin glargine from die diabetes registries DPV and DIVE. Wien Med Wochenschr. 2018;168:415-22.

32. Sullivan SD, Bailey TS, Roussel R, et al. Clinical outcomes in real-world patients with type 2 diabetes switching from first- to second-generation basal insulin analogues: comparative effectiveness of insulin glargine 300 units $/ \mathrm{mL}$ and insulin degludec in the DELIVER D+ cohort study. Diabetes Obes Metab. 2018;20:2148-58.

33. Ito H, Tsugami E, Ando S, et al. A comparison of the clinical courses of type 2 diabetic patients whose basal insulin preparation was replaced from insulin glargine 100 units/mL to insulin glargine biosimilar or 300 units/mL: a propensity score-matched observation study. J Drug Assess. 2018;7:54-60.

34. Mauricio D, Meneghini L, Seufert J, et al. Glycaemic control and hypoglycaemia burden in patients with type 2 diabetes initiating basal insulin in Europe and the USA. Diabetes Obes Metab. 2017;19: 1155-64.

35. Ji L, Bonnet F, Charbonnel B, et al. Towards an improved global understanding of treatment and outcomes in people with type 2 diabetes: rationale and methods of the DISCOVER observational study program. J Diabetes Complications. 2017;31: 1188-96.

36. Freemantle N, Bonadonna RC, Gourdy $\mathrm{P}$, et al. Rationale and methodology for a European pooled analysis of postmarketing interventional and observational studies of insulin glargine $300 \mathrm{U} / \mathrm{mL}$ in diabetes: protocol of REALI project. BMJ Open. 2020;10:e033659. 